\title{
单壁碳纳米管的控制生长与生长动力学
}

杨金龙

中国科学技术大学化学与材料科学学院, 合肥 230026

\section{Growth Kinetics and Structure Controlled Synthesis of Single-Walled Carbon Nanotubes}

\section{Jinlong Yang}

School of Chemistry and Materials Science, University of Science and Technology of China, Hefei 230026, P. R. China.

Email: jlyang@ustc.edu.cn.

Published online: February 17, 2020.

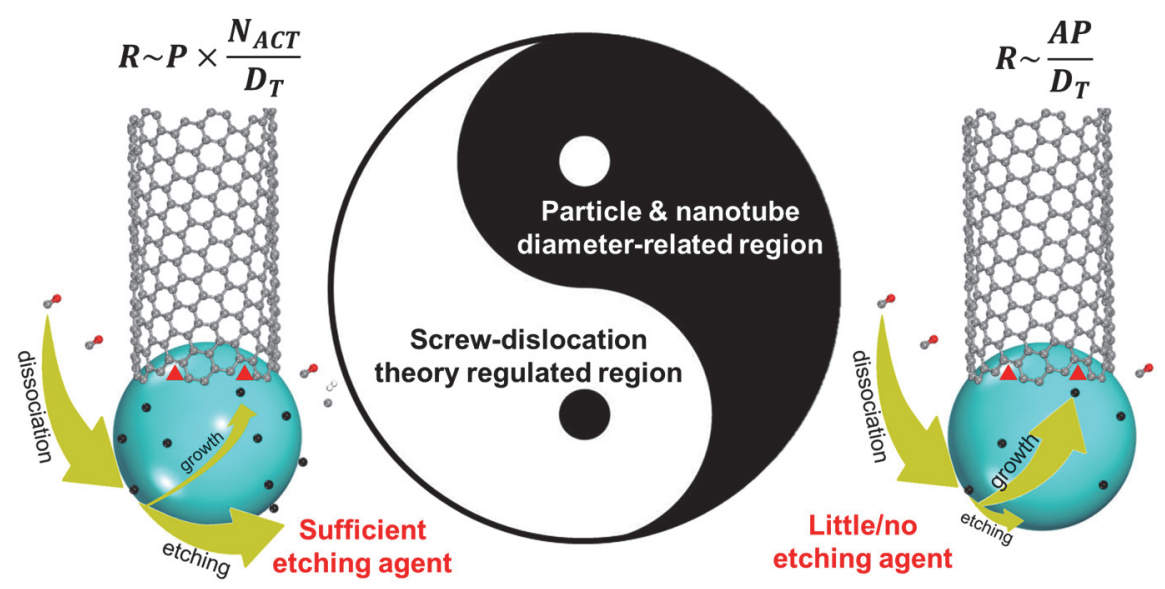

单壁碳纳米管在不同反应条件下的生长动力学。

结构决定性能, 作为典型一维碳材料的单壁 碳纳米管具有非常独特的光电性质, 因而, 单壁碳 纳米管的结构控制制备一直是人们关注的热点问 题, 也成为该领域最具挑战的课题之一 1,2 。目前, 化学气相沉积方法是可控制备碳纳米管的主要方 法, 在化学气相沉积反应过程中, 碳源在催化剂表 面裂解成核, 进而生长出结构不同、长度各异的单 壁碳纳米管。为深入探索碳纳米管的生长规律, 并 进一步提出更加合理的可控制备策略, 科学工作 者们长期致力于构建碳纳米管成核热力学和生长 动力学的理论框架。

在碳纳米管成核热力学方面, 特定结构的固 体催化剂已经被研究者们证实能够为碳纳米管的
成核提供更加稳定的模板, 并且影响其成核类型 3,4 。 例如, 2014年, 北京大学李彦等人利用高熔点的 $\mathrm{W}_{6} \mathrm{Co}_{7}$ 首次实现了 $(12 ， 6)$ 管的高纯度制备 ${ }^{3}$ 。 2017 年, 北京大学张锦等人则采用固体碳化钼和碳化 铇催化剂分别实现了高密度 $(12,6)$ 和 $(8,4)$ 碳纳米 管水平阵列的控制制备, 并揭示了固体催化剂晶 面与碳纳米管间的对称性匹配规律 ${ }^{4}$ 。2019年，该 研究组通过对碳纳米管成核热力学的进一步分 析, 实现了三重对称的 $(n, n-1)$ 型碳纳米管的富集 生长 ${ }^{5}$ 。同时在低温生长条件下, 通过控制生长条 件, 实现了六重(或三重)对称的(12，6)或三重对 称的 $(10 ， 9)$ 等碳纳米管的富集生长 ${ }^{6}$ 。

尽管研究者们在碳纳米管的成核热力学方面 
取得了巨大进步, 却往往忽视了生长动力学在其 生长过程中的重要作用。目前碳纳米管的生长动 力学研究主要集中在理论层面, 而实验上的进展 则远远滞后。早在2009年, 丁峰等人率先提出了螺 旋位错理论 7 。他们指出, 碳纳米管的生长速率与 碳纳米管开口端的活性位点数成正比, 即单根碳 纳米管的生长速率或长度将仅仅取决于碳纳米管 的几何结构或手性。这一结论与许多实验结果相 吻合。然而, 一方面, 最新的一些实验结果表明, 螺旋位错理论并不是普适的, 即测定的单壁碳纳 米管的长度和手性角并没有明显关联 8,9 。另一方 面, 容易被忽视的是热力学稳定成核通常意味着 较慢的生长速率, 但是已经报道实现的 $(12,6)$ 和 $(8,4)$ 碳纳米管的富集生长都是在碳源浓度较大 (往往意味着生长速率较快)的环境下实现的, 这与 理想的热力学稳定成核机理有一些相悖 4 。

为解释这一实验现象, 青岛科技大学何茂帅 研究组与北京大学张锦研究组以及韩国蔚山国家 科学技术研究院丁峰研究组一起, 提出了一个更 加完善的碳纳米管生长动力学模型 10 。在新的模型 中, 他们以催化剂表面碳原子浓度为研究对象, 重 点讨论了碳浓度随反应体系中碳的供给、刻蚀剂 浓度以及碳纳米管开口端的活性位点数的关系, 阐 明了能够把催化剂表面的碳原子带出反应体系的 刻蚀剂浓度对碳纳米管生长速率的影响。当体系 中刻蚀剂的浓度很高时, 催化剂表面吸附的碳原 子大多被刻蚀剂带出反应体系。在此反应区间内, 碳纳米管的生长速率正比于其开口端的活性位点 数, 即螺旋位错理论是成立的。同时, 利用氧化镁 负载的钴作为催化剂, 他们从实验上实现了大手 性角单壁碳纳米管的选择性生长。

另一方面，当反应体系中没有刻蚀剂或刻蚀 剂的浓度很低时, 催化剂表面吸附的碳原子主要 被碳纳米管的生长所消耗。在此反应条件下, 碳纳 米管的生长速率不受其开口端的活性位点数的影 响, 而仅仅取决于催化剂的裸露表面积和碳纳米 管直径的比值。利用环境透射电镜, 研究者跟踪了 多根单壁碳纳米管的生长并计算了碳纳米管的生 长速率, 其实验结果与理论模型非常吻合 ${ }^{10}$ 。

基于这一新的生长机制, 研究者进一步提出
了控制碳纳米管生长的新途径一即在没有刻蚀性 气体的条件下, 通过增加碳源的供给能够选择性 地使活性位点数目少的碳纳米管的催化剂失活, 进 而终止这些碳纳米管的生长, 最终得到活性位点 数最多的碳纳米管的高选择性生长。他们的实验 结果也表明, 通过提高碳源的供给量, 在固态钴催 化剂表面可以实现纯度高达 $90 \%$ 的 $(2 n, n)$ 碳纳米 管的生长。

上述研究工作近期在 Science Advances 上在线 发表 ${ }^{10}$ 。此项工作不仅建立了新的碳纳米管生长动 力学模型, 完善了单壁碳纳米管的生长机制, 而且 为单壁碳纳米管的结构可控生长提供了新的思路。

\section{References}

(1) He, M.; Zhang, S.; Wu, Q.; Xue, H.; Xin, B.; Wang, D.; Zhang, J. Adv. Mater. 2019, 31, 1800805. doi: 10.1002/adma.201800805

(2) Wang, X.; He, M.; Ding, F. Mater. Today 2018, $21,845$. doi: 10.1016/j.mattod.2018.06.001

(3) Yang, F.; Wang, X.; Zhang, D.; Yang, J.; Luo, D.; Xu, Z.; Wei, J.; Wang, J. Q.; Peng, F.; Li, X.; et al. Nature 2014, 510, 522. doi: $10.1038 /$ nature 13434

(4) Zhang, S.; Kang, L.; Wang, X.; Tong, L.; Yang, L.; Wang, Z.; Qi, K.; Deng, S.; Li, Q.; Bai, X.; et al. Nature 2017, 543, 234. doi: $10.1038 /$ nature 21051

(5) Zhang, S.; Wang, X.; Yao, F.; He, M.; Lin, D.; Ma, H.; Sun, Y.; Zhao, Q.; Liu, K.; Ding, F.; Zhang, J. Chem 2019, 5, 1182. doi: 10.1016/j.chempr.2019.02.012

(6) Zhang, S.; Lin, D.; Liu, W.; Yu, Y.; Zhang, J. Small 2019, 1903896. doi: $10.1002 / \mathrm{smll} .201903896$

(7) Ding, F.; Harutyunyan, A. R.; Yakobson, B. I. Proc. Natl. Acad. Sci. USA 2009, 106, 2506. doi: 10.1073/pnas.0811946106

(8) Inoue, T.; Hasegawa, D.; Chiashi, S.; Maruyama, S. J. Mater. Chem. A 2015, 3, 15119. doi: 10.1039/C5TA02679B

(9) He, M.; Magnin, Y.; Amara, H.; Jiang, H.; Cui, H.; Fossard, F.; Castan, A.; Kauppinen, E.; Loiseau, A.; Bichara, C. Carbon 2017, 113, 231. doi: 10.1016/j.carbon.2016.11.057

(10) He, M.; Wang, X.; Zhang, S.; Jiang, H.; Cavalca, F.; Cui, H.; Wagner, J. B.; Hansen, T. W.; Kauppinen, E.; Zhang, J. Sci. Adv. 2019, 5, eaav9668. doi: 10.1126/sciadv.aav9668 